\title{
MAXIMUM ENTROPY OPTIMUM DESIGN FOR PRODUCT STRUCTURE
}

\author{
Xu Yang ${ }^{1}$, Xingyuan Wang ${ }^{2}$ \\ 'Shandong University of Architecture and Engineering, China; Email: yangxu@sdai.edu.cn. \\ ${ }^{2}$ Shandong University, China.
}

\begin{abstract}
Taking the product cost as goal function, a maximum entropy method is used to set up a mathematical model for mechanical structural optimization. By using MATLAB, the computing program is made. And then an example for a mechanical product is given out - a motorcycle frame optimum, this is an optimization with sizes, stresses and frequency constraints. Results show that the optimum plan is feasible and effective in saving cost.
\end{abstract}

Key words: Maximum Entropy; Structure Optimization; Motorcycle Frame; Cost Reduction.

\section{INTRODUCTION}

With the development of modern science and technology, the market competition has become more and more acute. How to talent show itself in many products with the same function and the quality, on the one hand, the mechanical product structure is required to be reasonable, the function is comprehensive and the quality be excellent, on the other hand, the relevant cost be lower. Accordingly, the designer must do their best to meet many demands in the product function, and so on, and at the same time, to reduce cost as fully as possible as. The contradictious problem can be described as a mathematics model to be solved by the maximum entropy optimal method (MEOM).

Based on the surrogate constraint and the information entropy concepts, the iteration for the surrogate multipliers was set up [1], and all constraints in different forms were united handled [2], replaced by a very simple formulas and the algorithm is effective in the static strength problems of a truss [3].

Please use the following format when citing this chapter:

Yang, Xu, Wang, Xingyuan, 2006, in International Federation for Information Processing (IFIP), Volume 207, Knowledge Enterprise: Intelligent Strategies In Product Design, Manufacturing, and Management, eds. K. Wang, Kovacs G., Wozny M., Fang M.,

(Boston: Springer), pp. 192-197. 
The reference [4] introduces the convergence factors $\beta$ and $\gamma$ into the MEOM for the optimal design of rattled beam to force the design variables to get optimum. The convergence speed and the precision are improved obviously. In this paper, the author makes an optimal design example for a mechanical product-the lowest cost control of a motorcycle frame, which contains the safety and the comfortable constraint conditions.

\section{PRODUCT STRUCTURE OPTIMAL DESIGN}

To design a mechanical product which is composed of several parts with the same mechanical properties and the same price the target of the optimal design is to make the lowest cost, and satisfy the geometric size and the strength constraint conditions. Because the product has dynamic inputs, the natural frequency must escape the limiting frequency to prevent the resonance problem, by doing so, the safety and the economic properties of the product will be ensured.

Let: $\mathrm{U}$ denotes the cost; Fs denotes the surrogate constraint; $\mathrm{X}$ denotes the design variable. The optimization problem can be described as following:

$$
\begin{aligned}
\operatorname{Min} \quad U & =\sum_{i=1}^{n} C_{i} W_{i}(X) \\
\text { s.t. } \quad F_{s} & =\sum_{j=1}^{m} \lambda_{j} F_{j}(x) \leq 0 \quad \mathrm{i}=1,2, \ldots, \mathrm{n} ; \mathrm{j}=1,2, \ldots, \mathrm{m}
\end{aligned}
$$

Where: $\mathrm{Ci}, \mathrm{Wi}$ are the unit price and the weight of the $\mathrm{i}$-section separately, here the product is divided into $n$ parts, each part has a design variable; $\lambda \mathrm{j}$, $\mathrm{Fj}(\mathrm{x})$ are the surrogate multiplier and the constraint function of the $\mathrm{j}$-th constraint, there are $\mathrm{m}$ different constraints.

From the point of view of the probability [5], the $\lambda$ satisfies:

$$
\sum_{j=1}^{m} \lambda_{j}=1, \lambda_{j} \geq 0, \mathrm{j}=1,2, \ldots, \mathrm{m}
$$

Its value can be determined by: $\lambda_{j}=\exp \left[\theta F_{j}(X)\right] / \sum_{j=1}^{m} \exp \left[\theta F_{j}(X)\right]$

Where $\theta$ is a small positive number, let: $\theta_{j+1}=\theta_{j}+10^{-3}$.

By introducing the Lagrange multiplier $\alpha$ for Fs, we structure the Lagrange function: $L_{s}(X, \alpha)=U(X)+\alpha \cdot F_{s}(X)$

From the stationary conditions, that is: $\partial L_{s} / \partial x_{i}=0, \partial L_{s} / \partial \alpha=0$.

We can obtain: $\quad X_{k+1, i}=X\left\lfloor X_{k, i}, \lambda, \alpha\right\rfloor_{\mathrm{k}=1,2, \ldots, \mathrm{K}}$ 
Where $\mathrm{K}$ is the iteration times. It goes without saying that the possible paths exploring for the lowest cost is an iteration process, first, a group of initial value $X_{i}^{0}$ are given; then start to iterate and get a new group of design variable $X_{i}^{k}$, repeating this way, until the results satisfy the convergence criterions.

\section{ENGINEERING EXAMPLE}

\subsection{Vibration test}

Two-wheel-treadle motorcycle mainly drives in the city street, the speed of design in common use is the $30 \mathrm{Km} / \mathrm{h}$. driving in this speed, and the driver fell the vibration is rather greatly ${ }^{[6]}$. To explore the reasons of vibration, and adopt the measure, do two experiment researches as follows.

\subsubsection{Road-driving vibration test}

The measuring points of whole motorcycle are: Handlebar, Back-wheel axle, Engine in fixed-point of the frame, Back-vibration isolator in fixedpoint of the frame, Treadle, Saddle in three directions. The results (take measuring point on Treadle as example) are shown in Fig. 1.

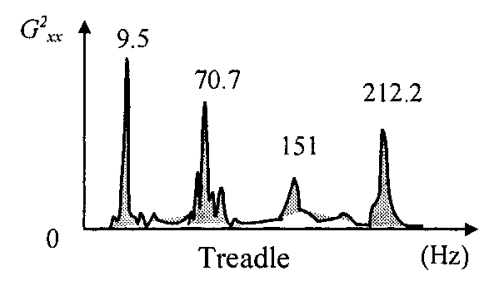

Figure 1. Vibratory frequency spectrum on line testing

Moreover, make the motorcycle rest in the laboratory, open the engine, test the vibration of each measuring point when the engine affect alone, that results and those on line test are similar, this test did not give.

\subsubsection{Experimental modal analysis of a frame ${ }^{[7]}$}

A motorcycle-frame structure is shown in Fig. 2. Its model parameters are shown in Tab.1. 


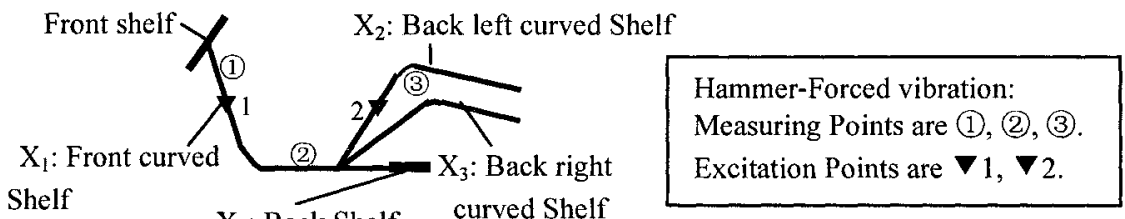

Figure 2. Motorcycle-frame Structure

Table 1. The frame model parameters

\begin{tabular}{llllll}
\hline Order.No. & 1 & 2 & 3 & 4 & 5 \\
\hline Model frequency(Hz) & 66 & 151 & 185 & 258 & 284 \\
Damping ratio (\%) & 1.515 & 0.662 & 0.541 & 0.386 & 0.335 \\
\hline
\end{tabular}

From above results analysis, we know: That power spectrum curve has a resonance peak in low frequency $9.5 \mathrm{~Hz}$ or so, the peak is a vibration owing to road excitation to a frame, and it can be neglected. The engine is the most important vibration source of the motorcycle. Designing a frame structure again, make its natural frequency keep away excitation frequency, is one of the necessary measures for raising the driver comfort and safety.

\subsection{Optimal model ${ }^{[8]}$}

The frame is simplified to three-dimensional beam units, the quantity on each unit is divided equally in each node, and the moments of inertia also do the same processing. The Front shelf does not attend the optimal design, the finite element model: 40 units, 41 nodes. The finite element calculation results (the natural frequency) are: 66.8, 152.7, 192.6, 245.5, $285.9 \mathrm{~Hz}$. There's a bit difference between the calculation values and test results.

A motorcycle frame is a space structure; the optimal control of the cost is that the cross acreage will be selected to lighten the weight of the frame, and satisfy the stress, the size and the frequency constraint conditions under the actual loads.

The optimization problem can be indicated as follows:

$$
\begin{aligned}
& \operatorname{Min} \quad U=\sum_{i=1}^{n} C_{i} \rho_{i} L_{i} A_{i}=\sum_{i=1}^{n} C_{i} \rho_{i} L_{i} x_{i}^{-1} \\
& \text { s.t. } \quad F_{1}=\left(\omega_{1}^{2} / \underline{\omega}^{2}-1\right) \leq 0 ; F_{2}=\left(1-\omega_{2}^{2} / \bar{\omega}^{2}\right) \leq 0 \\
& F_{i+2}=\left(A_{\min }^{i} \cdot x_{i}-1\right) \leq 0 \\
& F_{s}=\lambda_{1} \cdot F_{1}+\lambda_{2} \cdot F_{2}+\sum_{i=1}^{n} \lambda_{i+2} \cdot F_{i+2} \leq 0, \mathrm{i}=1,2, \ldots, \mathrm{n}
\end{aligned}
$$


Where $\rho_{i}, L_{i}, A_{i}$ are the density, the length and the cross section of the $i$-th length acreage separately, using the reciprocal design variable $x_{i}\left(x_{i}=1 / A_{i}\right)$, the variable number is 4 (shown in Fig.2.), $\omega_{1}, \omega_{2}, \omega_{t}, \omega_{F}$, are the first, the second eigenvalue and its lower, the higher limit of the restricted bounds; $A_{\text {min }}^{i}$ is the lower limit of $i$-th part cross section acreage constraints. The equation (8) satisfies not only the demand of the strength, but also the technology and the structure.

The frequency gradient formula:

$$
\partial \omega_{p}^{2} / x_{i}=\frac{\left\{U_{p}\right\} \frac{\partial K}{\partial x_{i}}\left\{U_{p}\right\}-\omega_{p}^{2}\left\{U_{p}\right\}^{T} \frac{\partial M}{\partial x_{i}}\left\{U_{p}\right\}}{\left\{U_{p}\right\}^{T} M\left\{U_{p}\right\}}
$$

Where $M$ and $K$ are the mass and stiffness matrices, let $\psi$ denotes the quadratic moment of per unit acreage, then the moment of inertia of the cross section of the frame is: $I_{i}=\psi A_{i}^{2}$, which will be used in the finite element analysis of the structure. The optimal analysis steps are shown in figure 4. In Fig.4, $\beta(0<\beta<1)$ and $\gamma(0<\gamma<1)$ are the geometric and the dynamic convergence factors. Let: $A_{\min }^{i}=\{0.27,0.15,0.15,0.25\} \mathrm{cm}^{2}$; Modulus of elasticity $E=210 \mathrm{GPa} ; \quad C=6000 \mathrm{yuan} / T ; \quad \omega_{\text {down }}=458 \mathrm{rad} / \mathrm{s}$, $\omega_{u p}=955 \mathrm{rad} / \mathrm{s}$; The natural frequency $f_{I}=67 \mathrm{~Hz}, f_{2}=152 \mathrm{~Hz}, \omega=2 \pi f$. The optimal results of a motorcycle frame are shown In Tab.2, and Tab.3.

Table 2. The optimal results

\begin{tabular}{lllll}
\hline Plan.No. & Ai $(\mathrm{cm} 2)$ & W $(\mathrm{kg})$ & Reduced-W $(\mathrm{kg})$ & $\mathrm{f1}, \mathrm{f2}(\mathrm{Hz})$ \\
\hline Original & $\{333.8,216.0,216.0,575.0\}$ & 4.443 & & $66.83,152.7$ \\
Plan 1 & $\{292.4,192.1,192.1,538.6\}$ & 3.886 & 0.557 & $71.24,6.73$ \\
Plan 2 & $\{275.1,200.3,200.3,538.6\}$ & 3.891 & 0.552 & $71.29,6.83$ \\
Plan 3 & $\{294.5,192.1,192.1,653.8\}$ & 3.929 & 0.514 & $70.86,5.89$ \\
Plan 4 & $\{310.2,200.1,200.1,596.4\}$ & 3.938 & 0.505 & $72.04,158.43$ \\
Plan 5 & $\{276.4,200.2,200.2,596.4\}$ & 3.916 & 0.527 & $71.41,56.63$ \\
\hline
\end{tabular}

\subsection{Analysis}

(1) In Each optimum process, the superior local value can be obtained through 4-6 iterations.

(2) The geometric convergence factor $\beta$ affected the convergence speed, and the frequency convergence factor $\gamma$ affected the precision, if its value is selected improper, the iteration will be difficult to converge even more.

Table 3. The saving cost per motorcycle

\begin{tabular}{lllllll}
\hline Plan. No. & 1 & 2 & 3 & 4 & 5 \\
\hline Save cost (Yuan) & 3.34 & 3.32 & 3.08 & 3.04 & 3.16 \\
\hline Rate of Reduced Cost & $12.5 \%$ & $12.4 \%$ & $11.6 \%$ & $11.3 \%$ & $11.8 \%$ \\
\hline
\end{tabular}




\section{CONCLUSIONS}

(1) The frequency constraint is a non-linear function which it hidden the design variables. So, for obtaining the global optimum solution, we can select several representative groups of design variables as the initial values to calculate the optimal problem, generally speaking, each optimal result is the local solution, we may consider the there exists global optimization value among these. Above the process can be called the first-stage optimization process.

(2) The Second-stage optimization process ${ }^{[9]}$ : The motorcycle frame uses the steel tube Structure, after determining the cross section acreage, many different standards can be selected, so the optimal design for the product structure will be obtained.

(3) Form table.3, we know: each motorcycle can be saved 1.6 Yuan on cost equally. In 2001, the manufacturer that the motorcycle discussed had obtained the highest sales volume (about a million motorcycle), if used the paper's design, they will save several millions Yuan; the economic benefit will rise greatly.

\section{REFERENCES}

1. E T Jaynes, (1957), Information Theory and Statistical Mechanics. The Physical Review, Vol. 106, pp. 620-630.

2. Templeman, A. B, Li Xingsi, (1987), A Maximum Entropy Approach to Constrained NonLinear Programming. Engineering Optimization, No.3, pp. 191-205.

3. Li Xingsi, (1989), A Maximum Entropy Method for the Structure Optimization Design, Computational Structural Mechanics and Application, No.1, pp. 36-45.

4. Yang Xu, (1996), A Maximum Entropy Method for the Dynamical Structural Optimization Design, Engineering Mechanics (Supplementary Issue), Vol.2, pp. 598-601.

5. C E Shannon. (1948), A Mathematical Theory of Communication, Bell System Technical Journal, Vol.27, No.3, pp. 379-428.

6. Shuqian Cao. Etc. (2001), Vibration Structure Modal Analysis - Theory, Experiment and Application, Tianjing University Press, Tianjing.

7. K. H. Hueber, (1975), The Finite Element Method for Engineers, John Wiley.

8. Edward J. Haug, Jasbir S. Arora. (1985), Applied Optimal Design, Science Press. Peking.

9. Teng Haiwen, (2002), Two-Stage Frame Structure Optimum Design with Discrete Variables Based on Maximum Entropy, Journal of Beijing Polytechnique University, Vol28, No.3, pp. 78-84. 